\title{
Disparity in Allosteric Interactions of Monastrol with Eg5 in the Presence of ADP and ATP: A Difference FT-IR Investigation ${ }^{\dagger}$
}

\author{
Edward J. Wojcik, $₫$ Nadine A. Dalrymple, ${ }^{\S}$ Shannon R. Alford, ${ }^{\S}$ Richard A. Walker $₫$ and Sunyoung Kim*, \\ Department of Biology and Department of Biochemistry, Virginia Polytechnic Institute and State University, \\ Blacksburg, Virginia 24061
}

Received May 19, 2004; Revised Manuscript Received June 23, 2004

\begin{abstract}
Eg5 is a kinesin-like motor protein required for mitotic progression in higher eukaryotes. It is thought to cross-link antiparallel microtubules, and provides a force required for the formation of a bipolar spindle. Monastrol causes the catastrophic collapse of the mitotic spindle through the allosteric inhibition of Eg5. Utilizing a truncated Eg5 protein, we employ difference infrared spectroscopy to probe structural changes that occur in the motor protein with monastrol in the presence of either ADP or ATP. Difference FT-IR spectra of Eg5-monastrol-nucleotide complexes demonstrate that there are triggered conformational changes corresponding to an interconversion of secondary structural elements in the motor upon interaction with nucleotides. Notably, conformational changes elicited in the presence of ADP are different from those in the presence of ATP. In Eg5-monastrol complexes, exchange of ADP is associated with a decrease in random structure and an increase in $\alpha$-helical content. In contrast, formation of the Eg5-monastrolATP complex is associated with a decrease in $\alpha$-helical content and a concomitant increase in $\beta$-sheet content. One or more carboxylic acid residues in Eg5 undergo unique changes when ATP, but not ADP, interacts with the motor domain in the presence of monastrol. This first direct dissection of inhibitorprotein interactions, using these methods, demonstrates a clear disparity in the structural consequences of monastrol in the presence of ADP versus ATP.
\end{abstract}

Inhibition of mitosis is at the crux of clinical strategies for controlling tumor growth. Mitotic motor proteins, including Eg5 ${ }^{1}$ dynein, and C-terminal kinesins, are required for bipolar spindle formation during mitosis. Small molecular inhibitors of the mitotic kinesin Eg5 have been uncovered from chemical screens; these are monastrol (1), terpendole E (2), and HR22C16 (3). These compounds arrest mitosis via reversible, allosteric inhibition of Eg5 and subsequent perturbation of bipolar mitotic spindle formation. It is noteworthy that these compounds do not affect other kinesin family members. Thus, inhibition of kinesin Eg5 provides a novel and specific mechanism for targeting the mitotic spindle, and a possible avenue for conferring general antiproliferative effects on cancerous growth.

$\mathrm{Eg} 5$ is a member of the $\mathrm{BimC} / \mathrm{Eg} 5$ (or N-2) class of the kinesin superfamily (4). The native Eg5 molecule is a homotetramer, organized with two sets of antiparallel dimers (5).

Supported by the OSER/Carilion Biomedical Institute (E.J.W. and S.K.), ASPIRES (S.K., R.A.W., and E.J.W.), NSF (Grant MCB0130910 to R.A.W.), and NIH (Grant GM066328 to E.J.W.).

* To whom correspondence should be addressed: 124 Engel Hall, West Campus Drive, Blacksburg, VA 24061. Phone: (540) 231-8636. Fax: (540) 231-9070. E-mail: sunyoung@vt.edu.

$\doteqdot$ Department of Biology.

$\S$ Department of Biochemistry.

${ }^{1}$ Abbreviations: AMP-PCP, adenosine $5^{\prime}$-( $\beta, \gamma$-methylene)triphosphate; DMSO, dimethyl sulfoxide; Eg5, kinesin motor; caged ATP, 1-(2-nitrophenyl)ethyl adenosine 5'-triphosphate; caged ADP, 1-(2nitrophenyl)ethyl adenosine $5^{\prime}$-diphosphate; FT-IR, Fourier transform infrared; HEPES, 4-(2-hydroxyethyl)piperazine-1-ethanesulfonic acid; monastrol, 4-(3-hydroxyphenyl)-6-methyl-2-thioxo-1,2,4-trihydropyridine-5-carboxylic acid ethyl ester; NPE, 1-(2-nitrophenyl)ethyl; Tris, tris(hydroxymethyl)aminomethane.
The resulting pair of motor domains at each end of the tetrameric molecule are thought to cross-link microtubules in the mitotic spindle: the motor domains are attached to antiparallel microtubules and drive the separation of spindle poles by sliding microtubules against each other $(6,7)$. Kinesins share a mechanism of conformational "switching" for converting small structural changes in their nucleotidebinding sites into larger movements to provide force generation and motion. Responsible for microtubule binding and force generation, the motor domain of kinesins also implements a nucleotide (ATP) hydrolysis cycle. Major transitions in the nucleotidase cycle are (1) binding of NTP, (2) bond cleavage (protein-NTP to protein $-\mathrm{NDP}-\mathrm{P}_{\mathrm{i}}$ ), (3) phosphate release (protein $-\mathrm{NDP}-\mathrm{P}_{\mathrm{i}}$ to protein $-\mathrm{NDP}$ ), and (4) NDP release (protein-NDP to protein and NDP).

Several techniques have provided information concerning the structural mechanotransduction of kinesins, including, for example, X-ray crystallography $(8,9)$ and magnetic resonance spectroscopy (10). The crystal structure of a truncated Eg5 with MgADP bound (8) has been determined; its overall folding pattern of a central eight-stranded $\beta$-sheet, with three helices on each side, is similar to that of other kinesins. Despite insight gained from structural studies utilizing nonhydrolyzable nucleotide analogues, detailed models of both the nucleotidase and mechanotransduction cycles, including a description of protonation and hydrogen bonding interactions of amino acid side chains, have not been determined. Elucidation of these mechanisms has been hampered by the lack of a complete set of structural data throughout a nucleotidase cycle for motor proteins or for structurally related G-proteins. However, structural similari- 
<smiles>CCOC(=O)C1=C(C)NC(=S)NC1c1cccc(O)c1</smiles><smiles></smiles><smiles>CC(=O)c1ccccc1N=O</smiles>

A

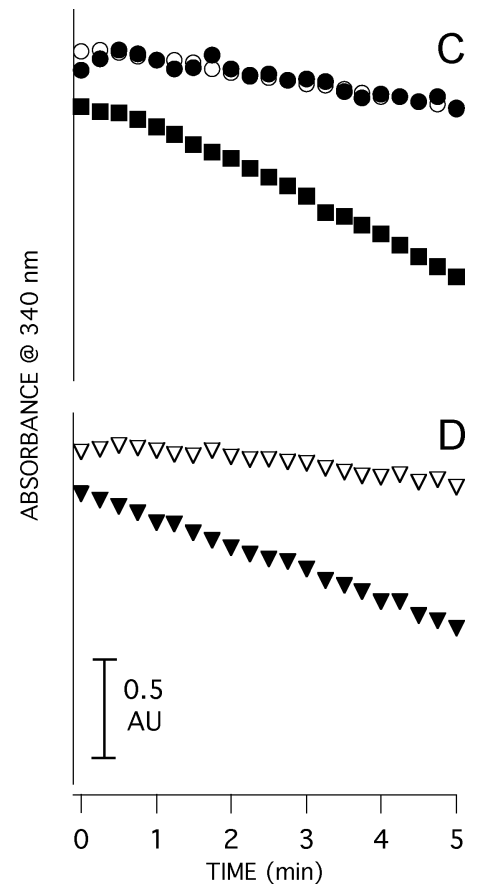

FIGURE 1: Structures of compounds and their effects on Eg5 activity. (A) Chemical structure of monastrol. (B) Schematic of the photolysis

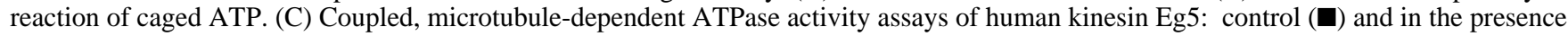
of monastrol (-). Also shown is the absorbance of monastrol in the absence of protein, under these assay conditions (O). The ATPase activity was $15 \pm 3,0.3 \pm 0.1$, and $0.3 \pm 0.1 \mathrm{~s}^{-1}$ for control Eg5, monastrol and Eg5, and monastrol alone, respectively. (D) Coupled, microtubule-dependent ATPase activity assays of human Eg5: with caged ATP $(\nabla)$ and with photolyzed caged ATP $(\nabla)$. The ATPase activity was $0.3 \pm 0.1 \mathrm{~s}^{-1}$ for Eg5 and caged ATP and $15 \pm 3 \mathrm{~s}^{-1}$ for Eg5 and photoreleased, caged ATP. Some data have been displaced along the $y$-axis to facilitate comparison. Data shown are averages of at least three independent experiments with two different protein preparations.

ties in the conformational switch between NTP and NDP states of motor proteins and G-proteins have helped identify key structural components of the nucleotidase cycle $(11,12)$. These include the conserved P loop, switch I, and switch II/helix regions that have been established as important motifs involved in critical changes in the nucleotidase cycle: the $\mathrm{P}$ loop is responsible for the binding of the $\alpha$ - and $\beta$-phosphate groups of the nucleotide, whereas switch I and switch II loops interact selectively with the $\gamma$-phosphate moiety.

The emergence of small-molecule inhibitors, which allosterically block the activity of mitotic motors, has enhanced the need for elucidation of the residues and regions of these proteins which trigger critical changes in the nucleotidase cycle. Monastrol, a dihydropyrimidine derivative (Figure 1A), provides us with a tool that may allow dissection of structural changes that may occur in Eg5. Irrespective of the presence of microtubules, monastrol allosterically inhibits ATP hydrolysis in Eg5 and a subset of homologues, but not other members of the kinesin and dynein superfamilies (13, 14). It causes reversible binding of $\mathrm{Eg} 5$ to microtubules that is resistant to both high and low concentrations of ATP and/ or salt $(13,14)$, conditions that cause the release of kinesinlike proteins from microtubules. Importantly, binding of monastrol in the presence of MgADP triggers widespread conformational changes throughout the Eg5 catalytic domain, possibly resulting in the arrest of Eg5 motor activity (9). However, the effects of monastrol on the core catalytic domain during nucleotide hydrolysis are unclear. Further dissection of the mechanism of allosteric inhibition by monastrol on Eg5 is crucial to understanding mechanotransduction and clarifying its potential as an anticancer agent.
Difference infrared spectroscopy permits observation of the structural changes that occur in Eg5 and monastrol during its nucleotidase cycle. In principle, vibrational spectroscopy can provide information about bond orders, charge distribution, and hydrogen bonding of individual groups. Dynamic and reversible changes in a single amino acid within multisubunit complexes of $>300 \mathrm{kDa}$ can be monitored by difference FT-IR (15), using small quantities of sample in solution and at room temperature. Difference (reactioninduced) vibrational spectroscopy offers the possibility of following changes concomitantly in the structure of Eg5, nucleotide, and monastrol in native conformations, instead of under specific forced conformations stabilized within crystals. Dynamic structural information uncovered by difference FT-IR can be combined with static structural information from X-ray crystallographic studies to produce a detailed model of Eg5 in mechanotransduction and inhibition.

Our aim in this vibrational study is to obtain direct experimental observation of structural changes in human Eg5, modulated by monastrol and the nucleotide substrate. Our strategy involves the incubation of active Eg5 motor domain and monastrol with "caged" ATP or ADP (16), which is used as a photolabile trigger to permit ternary interactions during spectroscopic observation. For example, the molecular cage $\left\{P^{3}\right.$-[1-(2-nitrophenyl)ethyl] or NPE $\}$ is an ester derivative of ATP (Figure 1B), which sterically prevents ATP from interacting with the motor protein, and which can be released by flash photolysis. Difference infrared data presented herein demonstrate that formation of the Eg5-monastrol-ATP complex triggers conformational changes throughout the $\mathrm{Eg} 5$ 
catalytic domain, which differ significantly from those observed in the presence of ADP. The chemical nature of the bound nucleotide modifies the structural consequences in the Eg5-monastrol-nucleotide complex in vitro.

\section{EXPERIMENTAL PROCEDURES}

Protein Expression and Purification. The truncated version of Eg5 was generated by amplifying a portion of the coding sequence from human pancreatic adenocarcinoma cDNA IMAGE clone 2323202 (Research Genetics/Invitrogen, Carlsbad, CA) by PCR using Pfu Turbo polymerase (Stratagene, La Jolla, CA). Restriction sites were engineered into each PCR fragment by base substitution in primers. The truncated Eg5 cDNA, encoding residues 1-370, was subcloned into the pET 21d plasmid (Novagen, San Diego, CA) between restriction sites $\mathrm{NcoI}$ and $\mathrm{XhoI}$ in frame with a C-terminal six-His tag.

The truncated form of human kinesin Eg5 was expressed in Escherichia coli BL21(DE3)pLysS cells that were transformed with the above pET construct in $2 \times$ YT medium with appropriate antibiotics. Expression was induced with $1 \mathrm{mM}$ isopropyl 1-thio- $\beta$-D-galactopyranoside when cultures reached an $\mathrm{OD}_{600}$ between 0.5 and 0.75 . Cells were harvested after growing for $4 \mathrm{~h}$ at $30{ }^{\circ} \mathrm{C}$, resuspended in $50 \mathrm{mM}$ Tris- $\mathrm{HCl}$ (pH 8), $0.4 \mathrm{M} \mathrm{NaCl}, 1 \mathrm{mM}$ ethylenediamine- $N, N, N^{\prime}, N^{\prime}-$ tetraacetic acid, and $1 \mathrm{mM}$ phenylmethanesulfonyl fluoride at a ratio of $4 \mathrm{~mL}$ of buffer per gram of pellet, and frozen at $-80{ }^{\circ} \mathrm{C}$ until they were used. Upon thawing of the sample additional protease inhibitor cocktail (Sigma, St. Louis, MO), $2 \mathrm{mM} \mathrm{MgATP}$, and $0.1 \mathrm{mg} / \mathrm{mL}$ DNase I were added to the cell suspension, and the mixture was allowed to incubate for $30 \mathrm{~min}$ at room temperature. Subsequently, cells were lysed with a French press, and clarified by centrifugation at $12000 \mathrm{~g}$ for $45 \mathrm{~min}$.

The supernatant was applied to a $\mathrm{Ni}$ affinity column (Qiagen, Valencia, CA), washed with $50 \mathrm{mM}$ HEPES (pH 7.5), $75 \mathrm{mM} \mathrm{NaCl}$, and $10 \mathrm{mM}$ imidazole, and eluted with linear gradient from 10 to $150 \mathrm{mM}$ imidazole. Pooled fractions were subjected to subsequent desalting in $50 \mathrm{mM}$ HEPES (pH 7.5) and $10 \mathrm{mM} \mathrm{NaCl}$, and cation exchange chromatography (linear gradient from 10 to $500 \mathrm{mM} \mathrm{NaCl}$ ). The final eluate was desalted and concentrated using Centricon filter devices (Millipore, Bedford, MA). The protein was stored at approximately $0.5 \mathrm{mM}$ in $50 \mathrm{mM}$ HEPES ( $\mathrm{pH}$ 7.5), $50 \mathrm{mM} \mathrm{NaCl}$, and $20 \%$ glycerol at $-80{ }^{\circ} \mathrm{C}$ until it was used. The final protein is estimated to be $>90 \%$ pure, based on SDS-polyacrylamide electrophoresis, a noninterfering protein assay (Genotechnology, St. Louis, MO), and densitometry; the final protein yield is approximately $1 \mathrm{mg}$ of Eg5 per liter of bacterial culture. Microtubule-stimulated ATPase assays (17) confirmed that the preparation was active (see Results).

Infrared Spectroscopy. Prior to spectroscopic use, glycerol was removed from the Eg5 protein samples by dilution and concentration using Centricon filter devices. Infrared samples (total volume of $20 \mu \mathrm{L}$ ) contained $20 \mathrm{mM}$ HEPES (pH 7.5), $0.1 \mathrm{mM}$ EDTA, $6 \mathrm{mM}$ dithiothreitol, $1 \mathrm{mM} \mathrm{MgCl}, 10 \mathrm{mM}$ caged nucleotide, $1 \mathrm{mM} \mathrm{Eg} 5$ protein, and $1.25 \%$ DMSO (unless indicated as omitted); dithiothreitol is utilized to scavenge the reactive photolysis byproduct, 2-nitrosoacetophenone (Figure 1B). All experimental manipulations of caged ATP and ADP (Molecular Probes, 98-99\% pure), as well as the ${ }^{15} \mathrm{~N}$-nitro isotopomers of caged ATP, were conducted in the dark. Monastrol (Sigma) was incubated with $\mathrm{Eg} 5$, at a final concentration of $5 \mathrm{mM}$, for $10 \mathrm{~min}$ prior to the addition of the caged nucleotide to the IR samples.

To minimize the contributions of water to the FT-IR spectra, samples were partially dehydrated under a dry $\mathrm{N}_{2}$ stream (15). Reagent mixtures were subsequently sandwiched between two $\mathrm{CaF}_{2}$ windows. Samples, held fixed in the spectrometer with a demountable liquid cell holder (Harrick Scientific, Ossining, NY), were placed under vacuum for 1 $\mathrm{h}$ before data acquisition. Spectral conditions were as follows: double-sided, forward-backward acquisition mode, Happ-Genzel apodization function, $20 \mathrm{kHz}$ mirror velocity, $4 \mathrm{~cm}^{-1}$ spectral resolution, 200 scans per interferogram, 1 level of zero filling, gain of 1 , and room temperature. The duration for the acquisition of one interferogram was $86 \mathrm{~s}$.

A Nd:YAG Surelite Continuum (Santa Clara, CA) laser with $355 \mathrm{~nm}$ excitation was utilized for photolysis of caged nucleotides in either the absence or presence of Eg5. The laser energy output was $\sim 160 \mathrm{~mJ}$ per flash, with a pulse duration of 6 ns. Conversion of caged ATP was confirmed by HPLC analysis using a reverse-phase C-18 column under ion pairing conditions with $50 \mathrm{mM}$ potassium phosphate $(\mathrm{pH}$ 6.5), $5 \mathrm{mM}$ tetrabutylammonium bromide, and $20 \%$ acetonitrile. The absorbance change of the marker bands at 1526 and $1346 \mathrm{~cm}^{-1}$ was plotted against the number of flashes, and the saturation curve was normalized against the HPLC quantitation. Five flashes yielded 3-5\% conversion of total caged ATP to free ATP, concentrated in the fraction of nucleotide within the IR beampath. To ensure consistent release of the caged nucleotide, these conditions were held constant in all experiments.

Vibrational data were collected with an MCT/A detector in a Bruker IFS 66v/s infrared spectrometer under vacuum. All spectra shown herein are averages of at least three experiments, and were analyzed using IGOR Pro (Wavemetrics, Lake Oswego, OR). In our samples, the absorbance of all regions between 4000 and $900 \mathrm{~cm}^{-1}$ was less than 1 . For each sample, six interferograms were acquired prephotolysis (dark), followed by iterated collection of prephotolysis (dark) and postphotolysis (light) interferograms, sandwiching five successive laser flashes on the spectroscopic sample. The difference, or light-minus-dark, spectrum of the IR sample with the caged nucleotide was obtained by subtracting the spectrum after photolysis from the spectrum of the sample before illumination; six light-minus-dark and three darkminus-dark spectra were generated for each sample. Difference spectra are scaled to an absorbance of $0.3 \mathrm{AU}$, based on a $\Delta A$ between 1650 and $1800 \mathrm{~cm}^{-1}$. Reaction-induced data that are shown are averages of only the first light-minusdark spectra, which reflect steady-state conditions upon release of free nucleotide and from which the effects of NTP and NDP mixing can be excluded. Note that, as in other vibrational analyses of caged compounds $(16,18)$, construction of double-difference spectra utilizes an interactive subtraction to eliminate the 1525 and $1344 \mathrm{~cm}^{-1}$ lines, assigned to $\mathrm{NO}_{2}$ stretching vibrations, to remove contributions from the photolytic reaction. The ${ }^{15} \mathrm{~N}$-nitro isotopomers of caged ATP (19), generously provided by J. Corrie (National Institute for Medical Research, U.K.), downshift vibrational modes in the $1525 \mathrm{~cm}^{-1}$ region (see Results) and 
permit analysis of any potential protein bands in the 1525 $\mathrm{cm}^{-1}$ region (data not shown).

\section{RESULTS}

Interaction between Purified Eg5 and Caged Nucleotides. Our experimental model is the motor domain of the human Eg5 kinesin-like protein, which is a truncated form ( $42 \mathrm{kDa})$ of the wild-type protein that lacks an oligomerization domain. We tested the ability of the purified Eg5 motor domain to catalyze the hydrolysis of ATP using a coupled NADH activity assay, previously described (17); rates of this truncated Eg5 (Figure 1C) are comparable with those reported in the literature $(13,14)$. As expected, introduction of monastrol in the in vitro assay results in abolishment of activity (Figure 1C). Substitution of caged ATP in lieu of ATP in the assay mixture resulted in no measurable change in NADH absorption (Figure 1D). However, photolytic release of ATP from the nitrophenylethyl moiety permitted ATP hydrolysis with rates that paralleled those when ATP was used alone. Our data support the conclusion that caged ATP effectively sequesters ATP from the enzyme until the cage group is removed via UV photolysis.

Difference Infrared Spectrum of Caged Nucleotide Photolysis. Photolysis of caged nucleotides can be assessed with difference infrared techniques (Figure 2). To examine the spectrum of caged ATP photolysis, we collected FT-IR spectra of caged ATP before and after photolytic release of the NPE cage (Figure 2A, solid line). A difference spectrum results from the subtraction of a spectrum measured before photolysis of the NPE-caged ATP from a spectrum taken after photolysis. The control difference spectrum (Figure 2A, dotted line) shows the difference between two spectra measured before laser illumination; there are no observable spectral features in these data. Therefore, only those vibrational modes that undergo reaction-induced absorbance changes contribute to spectral features in the photolysis difference spectrum. Positive bands in the difference spectrum (Figure $2 \mathrm{~A}$, stippled shading) refer to new species produced by the photolysis reaction (Figure 1B), while negative bands (Figure $2 \mathrm{~A}$, solid shading) refer to the initial state of the educts.

Our data are consistent with vibrational data from other NPE-caged compounds (16). For example, the positive band at $1693 \mathrm{~cm}^{-1}$ (Figure 2A) is attributed to the nitrosoacetophenone (19). Negative bands at 1525 and $1344 \mathrm{~cm}^{-1}$ have been previously assigned to the symmetric and antisymmetric stretching vibrations of the disappearing $\mathrm{NO}_{2}$ group $(16,18)$. This is confirmed by use of ${ }^{15} \mathrm{~N}$-caged ATP (Figure 2B, solid line); this photolysis spectrum has spectral shifts (shaded negative lines) that arise from the isotopic substitution of the nitrophenylethyl moiety. The negative antisymmetric and symmetric $\mathrm{NO}_{2}$ vibrations of caged ATP are downshifted by 27 and $21 \mathrm{~cm}^{-1}$, respectively. In addition, the presence or absence (Figure 2A, dashed line) of low concentrations of DMSO, required for solubilizing monastrol, does not impact the photolysis spectrum.

The $1300-900 \mathrm{~cm}^{-1}$ region is dominated by changes in phosphate vibrations (Figure 2). At $\mathrm{pH} 7.5$, the $\mathrm{N} 1$ atom of the adenine ring and the $\gamma$-phosphate group of ATP are deprotonated; the triphosphate chain is composed of two $\mathrm{PO}_{2}{ }^{-}$groups at the $\alpha$ and $\beta$ positions, and a $\gamma \mathrm{PO}_{3}{ }^{2-}$ group. Antisymmetric stretching vibrations $\left(v_{\mathrm{a}}\right)$ of the $\mathrm{PO}_{2}{ }^{-}$groups are localized in the $1250-1150 \mathrm{~cm}^{-1}$ region (20). Sym-

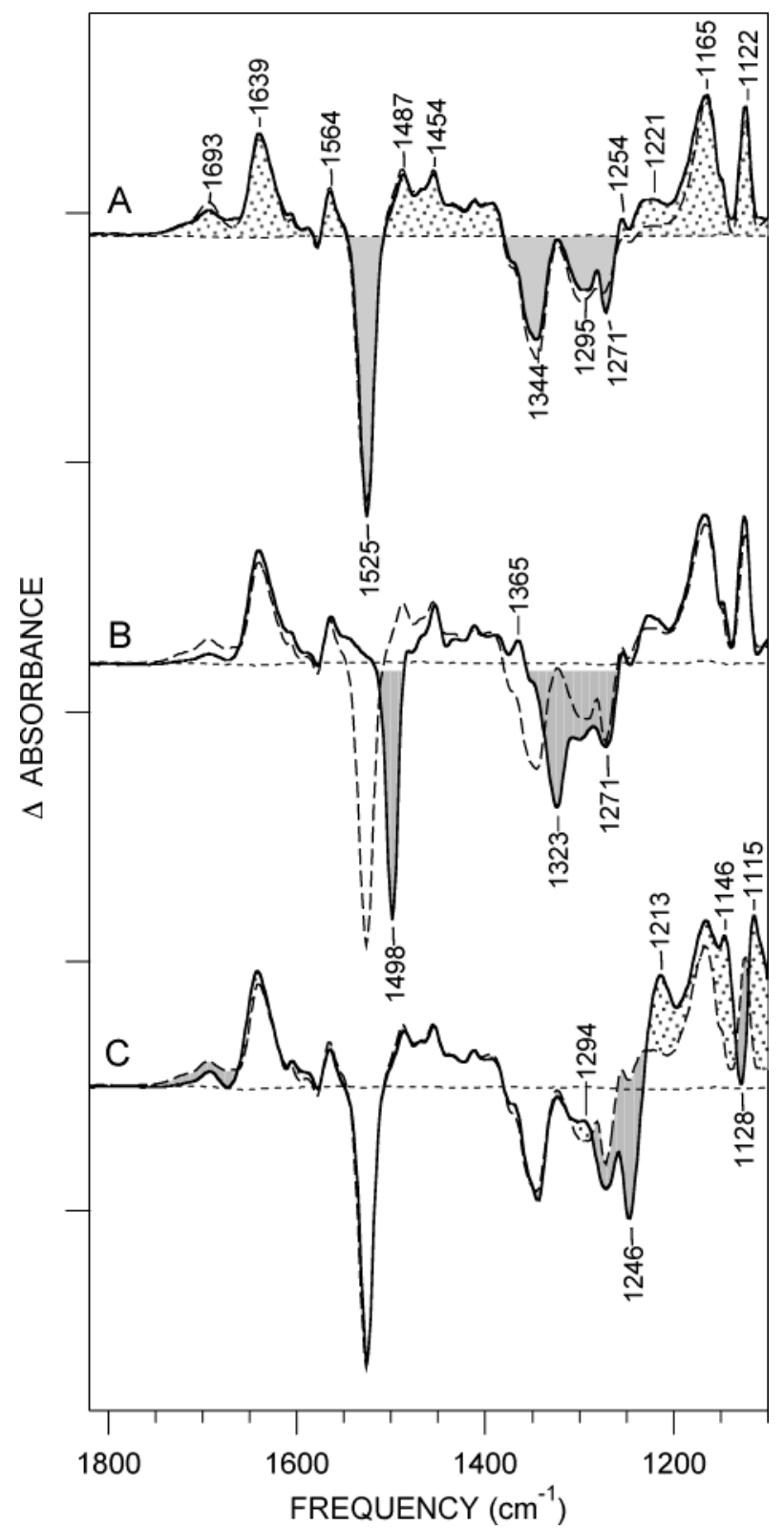

FIGURE 2: Difference infrared spectra of caged nucleotide photolysis. (A) Caged ATP photolysis in the presence $(-)$ and absence of DMSO (- - ). (B) Photolysis of ${ }^{15} \mathrm{~N}$-labeled caged ATP (-) in comparison with unlabeled caged ATP $(---)$. (C) Caged ADP photolysis (-) in comparison with caged ATP $(---)$. Control dark-minus-dark spectra, corresponding to the primary data in each panel, are shown in panels $\mathrm{A}-\mathrm{C}$ as dotted lines. Data shown in panels $\mathrm{B}$ and $\mathrm{C}$ were collected on samples containing DMSO, and are averages of 600-1200 infrared scans. Tick marks on the $y$-axis denote $1 \times 10^{-2} \mathrm{AU}$.

metric stretching vibrations of $\mathrm{PO}_{2}^{-}$, found in the $1100 \mathrm{~cm}^{-1}$ region, overlap with degenerate stretching modes of the $\mathrm{PO}_{3}{ }^{2-}$ terminal group expected in the $1120-1070 \mathrm{~cm}^{-1}$ region. Photolysis of caged ADP yields similar difference IR data (Figure 2C, solid line), with the exception of the $1300-1100 \mathrm{~cm}^{-1}$ region (shaded regions); the $v_{\text {as }}$ vibrations of the $\mathrm{PO}_{2}{ }^{-}$groups shift from $1230 \mathrm{~cm}^{-1}$ in ATP to 1210 $\mathrm{cm}^{-1}$ in $\operatorname{ADP}(20,21)$. As expected, there are no observed $\mathrm{HPO}_{4}{ }^{2-}$ vibrational modes (16) in the $1080-990 \mathrm{~cm}^{-1}$ region (data not shown).

Monastrol Binding Spectra of Eg5 and the Nucleotide. Likewise, we obtained a difference FT-IR spectrum of the photolysis of caged ATP in the presence of the Eg5 motor 


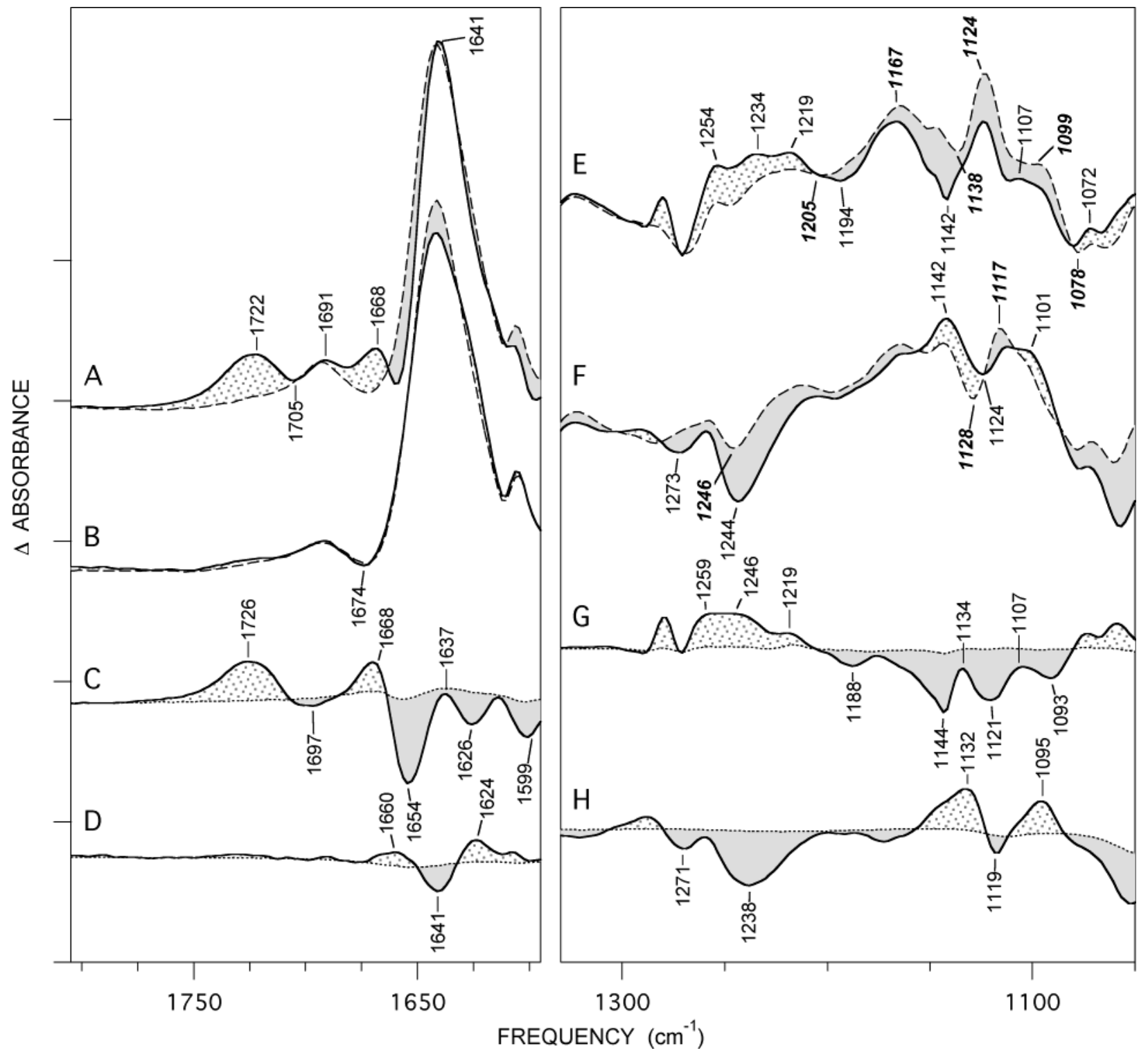

FIGURE 3: Difference infrared spectra of caged nucleotide photolysis in the presence of monastrol and Eg5. Shown is the $1800-1600 \mathrm{~cm}^{-1}$ region in panels A-D. (A) Caged ATP photolysis in the presence of Eg5. Monastrol was either preincubated with the sample ( - ), prior to photolysis, or absent $(---)$. (B) Caged ADP photolysis in the presence of Eg5. Monastrol was either preincubated with the sample $(-)$, prior to photolysis, or absent $(---)$. (C) Eg5-monastrol binding spectrum upon photolytic cleavage of caged ATP. This data (-) is a double-difference spectrum of Eg-monastrol-caged ATP minus Eg5-caged ATP. The control double-difference spectrum is shown as a dotted line. (D) Eg5-monastrol binding spectrum upon photolytic cleavage of caged ADP. This data ( - ) is a double-difference spectrum of Eg5-monastrol-caged ADP minus Eg5-caged ADP. The control double-difference spectrum is shown as a dotted line. Panels E-H depict the $1330-1050 \mathrm{~cm}^{-1}$ region of panels $\mathrm{A}-\mathrm{D}$, respectively. Tick marks on the $y$-axis denote $1.5 \times 10^{-3}$ and $8.8 \times 10^{-3}$ AU for the left and right panels, respectively.

domain and monastrol (Figure 3A, solid line). A second difference infrared spectrum was obtained for samples containing Eg5-ATP complexes in the absence of monastrol (Figure $3 \mathrm{~A}$, dashed line). Comparison of these difference infrared data shows clear spectral lines that are only evident when monastrol is present. In the $1800-1600 \mathrm{~cm}^{-1}$ region, which contains contributions from carbonyls, unique positive lines are observed at 1722 and $1668 \mathrm{~cm}^{-1}$ (Figure 3A, solid line) in the Eg5-monastrol-ATP spectrum. There are also frequency changes observed between 1655 and $1600 \mathrm{~cm}^{-1}$ that likely arise from carbonyl modes of the peptide backbone.

Difference FT-IR spectra of caged ADP photolysis in the presence of Eg5 and monastrol do not yield similar changes (Figure 3B, solid line) as observed in the presence of caged adenosine triphosphate. When compared to the control difference spectrum of caged ADP photolysis and Eg5
(Figure 3B, dashed line), these data exhibit a smaller number of changes in frequency and intensity. Notably, in the carbonyl infrared region, there are small changes in intensity at $1641 \mathrm{~cm}^{-1}$ (Figure 3B, solid line), and no observable unique positive modes at 1722 and $1668 \mathrm{~cm}^{-1}$ as seen for the Eg5-monastrol-ATP complex.

Double-difference spectra (Figure 3C,D) are constructed by subtraction of the difference FT-IR spectrum of caged nucleotide photolysis with Eg5 (Figure 3A,B, dashed lines) from the difference data of corresponding caged nucleotide release for the Eg5-monastrol complex (Figure 3A,B, solid lines). The resulting double-difference spectra are dominated by the effects of the nucleotide binding to $\mathrm{Eg} 5$ in the presence or absence of monastrol; these double-difference data represent monastrol binding spectra. Note that the negative controls (Figure 3C,D, dotted lines) do not exhibit significant 
spectral features. Therefore, we can measure dynamic structural changes in the motor protein that are inhibitor- and nucleotide-specific.

Carbonyl Region of the Monastrol Binding Spectra. Shown in Figure 3 is the spectral range that encompasses the absorption of the $\mathrm{C}=\mathrm{O}$ mode of carboxyl groups and of the amide I mode of the polypeptide backbone. The carbonyl stretching, or the amide I, vibration of the peptide backbone (22) dominates in the $1690-1620 \mathrm{~cm}^{-1}$ region of the monastrol binding spectra (Figure 3C,D, solid lines). Intensities and frequencies in this amide I region of the monastrol binding spectrum may be influenced by changes in secondary structure content or other changes in protein conformation upon nucleotide binding. Alternatively, a contribution in this region may be from the carbonyl group in monastrol or from side chain vibrations from primary amide groups such as glutamine and asparagine (23).

Data within the amide I region show that structural perturbations due to monastrol are not identical for ATP or ADP bound to the $\mathrm{Eg} 5$ motor domain. The monastrol binding spectrum in the presence of ATP (Figure 3C, solid line) exhibited negative spectral components at 1654 and $1626 \mathrm{~cm}^{-1}$, which were indicative of unique modes in the absence of monastrol. In addition, positive lines at 1668 and $1637 \mathrm{~cm}^{-1}$ were observed in this double-difference spectrum, arguing that the combined presence of monastrol and ATP yielded unique structural changes. Our data show that there are distinct differences in the $1690-1620 \mathrm{~cm}^{-1}$ region of the Eg5monastrol binding spectrum for caged ADP (Figure 3D, solid line); the absence of monastrol is correlated with a negative mode at $1641 \mathrm{~cm}^{-1}$, whereas small positive modes at 1660 and $1624 \mathrm{~cm}^{-1}$ are observed in the presence of monastrol. We conclude that, in solution, the motor domain of Eg5 can sample a variety of conformational states, which differ in their hydrogen bonding of the peptide backbone and which differ depending upon the available nucleotide.

Additionally, a striking feature can be seen in the $1750-1680 \mathrm{~cm}^{-1}$ infrared region: the only amino acid residues with fundamental vibrational transitions in the region are aspartic and glutamic acids (23), from which the carboxylic acid $\mathrm{C}=\mathrm{O}$ stretches generate modes with appropriate frequencies. In the Eg5-monastrol-ATP sample, observation of a broad, vibrational mode at $(+) 1726 \mathrm{~cm}^{-1}$ (Figure 3C) suggests that at least one aspartic or glutamic acid residue in $\mathrm{Eg} 5$ is perturbed. The frequency of the $\mathrm{C}=\mathrm{O}$ carboxylic acid stretches reflects changes in double bond character in the $\mathrm{C}=\mathrm{O}$ bond as well as alterations in the basicity of the oxygen $(23,24)$. Note that there is no corresponding mode in Eg5-monastrol-ADP samples (Figure $3 \mathrm{D}$, solid line). Calculated $\mathrm{C}=\mathrm{O}$ modes of monastrol are below $1700 \mathrm{~cm}^{-1}$ (D. Bevan, unpublished results). Therefore, we assign this $1726 \mathrm{~cm}^{-1}$ vibrational mode to the $\mathrm{C}=\mathrm{O}$ stretching vibration of a carboxylic acid, which is perturbed in human Eg5 upon formation of the Eg5 motor domain-monastrol-ATP complex.

Phosphate Region of the Monastrol Binding Spectra. Spectral signatures in the $1300-1000 \mathrm{~cm}^{-1}$ region of these biological difference data (Figure $3 \mathrm{E}-\mathrm{H}$ ) are attributed to changes in phosphate groups of nucleotides. However, spectral features observed in the presence of ATP and ADP are not identical within this region. Upon photolysis of caged ATP, there is a broad, positive mode between 1254 and 1219 $\mathrm{cm}^{-1}$, which is enhanced in the presence of monastrol (Figure 3E, stippled shading). Comparison of the $1167-1099 \mathrm{~cm}^{-1}$ spectral region in Figure 3E indicates that both decreased amplitude and vibrational shifts can be observed (solid shading) in the presence of monastrol. In contrast, photolysis of caged ADP in Eg5-monastrol samples yields an increased amplitude of negative modes in the $1260-1210 \mathrm{~cm}^{-1}$ spectral window (Figure $3 \mathrm{~F}$, solid shading) and an increased intensity concomitant with vibrational shifts in the $1167-1100 \mathrm{~cm}^{-1}$ region (stippled shading). The disparity of the resultant, spectroscopic modifications is highlighted in the monastrol binding spectra of caged ATP (Figure 3G) and caged ADP (Figure $3 \mathrm{H}$ ). Therefore, we detect interactions between Eg5 and the $\mathrm{PO}_{3}{ }^{2-}$ terminal group of these nucleotides. Furthermore, the lack of a strong positive mode at $\sim 1080 \mathrm{~cm}^{-1}$ indicates that there is no net, observed formation of $\mathrm{HPO}_{4}{ }^{2-}$ by Eg5, upon release of caged ATP.

\section{DISCUSSION}

To obtain novel molecular information about the inhibition mechanism of monastrol with human Eg5, we have employed reaction-induced FT-IR spectroscopy to study the interaction between monastrol, nucleotide, and protein. Vibrational spectroscopy can yield dynamic information about structural changes occurring in motor proteins. In this initial study, monastrol and the $\mathrm{Eg} 5$ motor domain are incubated in vitro, and caged ATP or caged ADP is then used as a photolabile trigger for the ternary interaction. Subtraction of caged nucleotide-protein contributions results in a double-difference spectrum corresponding to the unique structural changes arising solely from the presence of monastrol in the system. This report focuses on the first application of these techniques, which examine changes within $\mathrm{Eg} 5$ in the presence of ADP in comparison to those of ATP.

Perturbation of Phosphate Interactions with Eg5 in the Presence of Monastrol. Experimental debate about the mechanism for phosphoryl transfer reactions, such as ATP or GTP hydrolysis, includes associative, dissociative, concerted, and substrate-assisted arguments. Vibrational spectroscopy can provide detailed, mechanistically important information about the structures of substrates, cofactors, and even transition states, given an appropriate time resolution (for examples, see refs 25 and 26). A prerequisite of this complex, mechanistic dissection is analysis of a complete set of isotopomers: isotopic labeling allows clear assignment of vibrational lines to a chemical species, i.e., $\mathrm{PO}_{2}{ }^{-}$or $\mathrm{PO}_{3}{ }^{2-}$, and permits detailed examination of the structure of the species in question in conjunction with normal coordinate analysis. Although use of caged nucleotides was pioneered with the ATPase enzymes $(16,27)$, a larger set of data and highly developed analysis of phosphate modes exist for caged GTP $(25,26,28,29)$. Our interpretation of the phosphate region of the monastrol binding spectra (Figure $4 \mathrm{G}, \mathrm{H}$ ) is therefore based on an amalgam of work published using caged GTP and caged ATP.

Although our Eg5 motor protein is active in microtubulestimulated activity assays, we do not expect to observe marked ATP hydrolysis under our spectroscopic conditions. These difference infrared data were acquired in the absence of microtubules, and for Eg5 motor domains, basal rates of ATP hydrolysis are $\sim 200$-fold lower than those stimulated by microtubules $(13,14)$. Observation of $\mathrm{HPO}_{4}{ }^{2-}$ vibrational 

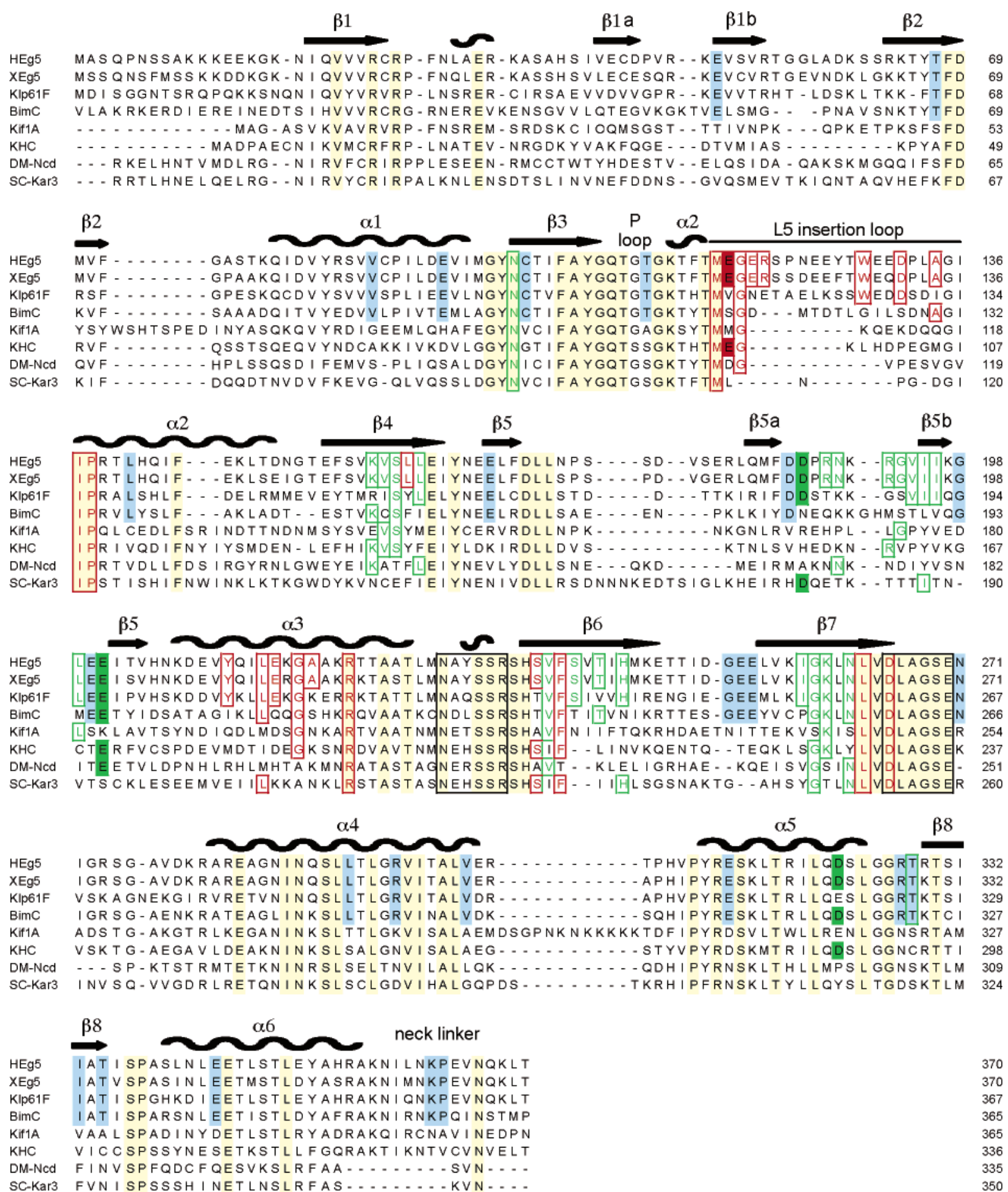

FIGURE 4: Analysis and identification of residues that line pocket surfaces for monastrol and ADP binding in Eg5. Shown is a CLUSTAL sequence alignment of human Eg5 (HEg5), Xenopus Eg5 (XEg5), a Drosophila Eg5 homologue (Klp61F), an Aspergillus Eg5 homologue (BimC), human monomeric kinesin (Kif1A), the human kinesin heavy chain (KHC), the Drosophila minus end-directed motor (Ncd), and the Saccharomyces Ncd homologue (KAR3), with appropriate notation of secondary structures found in human Eg5 (8). Conserved residues across all kinesins are shaded in light yellow, and those conserved within the Eg5 family (8) are shaded in light blue. Defined by PROCHECK, secondary structural elements are labeled, as defined for conventional kinesin (49). Switch 1 and switch 2 regions of Eg5 are boxed in black lines. Using CASTp (32), residues which contribute at least one atom to the surface of the ADP binding pocket were identified and are boxed in green. Amino acids which comprise the surface of the monastrol binding pocket were identified and are boxed in red. Candidate residues that are observed in the infrared data shown herein are highlighted with a red or green background, if found in the monastrol- or ADP-binding pocket, respectively.

modes is expected at 1068 and $992 \mathrm{~cm}^{-1}$ in solution (16), and at 1078 and $992 \mathrm{~cm}^{-1}$ in the presence of protein $(28)$. Upon photolytic release of caged ATP, we do not observe any evidence favoring the formation of orthophosphate, irrespective of monastrol binding (Figure 3G,H).
On the other hand, these Eg5-monastrol-ATP samples have changes in both frequency and intensity in the phosphate region. A broad series of lines are observed at 1259-1219 $\mathrm{cm}^{-1}$ in the presence of ATP and monastrol (Figure 3G, stippled shading). Protein interaction with ATP and GTP has 
been shown to shift frequencies of $\alpha$ and $\beta \mathrm{PO}_{2}{ }^{-}$moieties from $\sim 1230$ (20) to $1260-1219 \mathrm{~cm}^{-1}(21,25,28)$. Infrared lines of the terminal $\gamma \mathrm{PO}_{3}{ }^{2-}$ (16) between 1160 and 1100 $\mathrm{cm}^{-1}$ are shifted in frequency in the presence of monastrol (Figure 3G, solid shading). Examination of the ADP phosphate region also shows changes in vibrational modes in both frequency and intensity. The negative $1238 \mathrm{~cm}^{-1}$ line (Figure $3 \mathrm{H}$, solid shading) is, therefore, assigned to the asymmetric stretching mode $\left(v_{\mathrm{a}}\right)$ of $\alpha \mathrm{PO}_{2}{ }^{-}$when ADP is bound to protein $(21,28)$. Additional lines at 1130-1095 $\mathrm{cm}^{-1}$ in the monastrol binding spectrum for ADP (Figure $3 \mathrm{H})$ may arise from terminal $\beta \mathrm{PO}_{3}{ }^{2-}$. Confirmation of these tentative assignments requires isotopic labeling of the $\alpha$-, $\beta$-, and $\gamma$-phosphate groups $(25,28)$. Given the similarity to other published reports $(25,28)$, we speculate that these frequency shifts are likely to arise from variations in charge localization.

In summary, our infrared data do not support the argument that Eg5 hydrolyzes ATP in our spectroscopic experiments. This is consistent with the low overall ATPase rate of motors in the absence of polymer binding $(13,14,30)$. Therefore, one interpretation for the data herein is that structural changes observed with caged ATP result from the step in the nucleotidase cycle of NTP binding to the motor protein. In addition, the presence of monastrol in $\mathrm{Eg} 5$-nucleotide samples induces vibrational uncoupling of the $\mathrm{PO}_{2}{ }^{-}$moieties from the terminal $\mathrm{PO}_{3}{ }^{2-}$ group, and the terminal phosphate group has an asymmetric localization of charge that is perturbed in the presence of monastrol.

Carboxylic Acids Perturbed in the Monastrol-ATP-Eg5 Complex. The $1726 \mathrm{~cm}^{-1}$ absorbance that is observed only in the presence of both ATP and monastrol, but not notable in ADP and monastrol, is characteristic of carboxylates (Figure 3C). We assign this $1726 \mathrm{~cm}^{-1}$ line to a carboxylic acid in Eg5, as monastrol is theoretically not capable of generating such a mode. This is the first direct evidence of a structural change of a single amino acid in a motor protein, upon introduction of the native substrate, ATP. There are three categories of structural changes that would result in a carboxylic acid contribution to the vibrational monastrol binding spectrum of Eg5 and ATP. The first type of structural change is a modification of the protonation state of the carboxylic acid. The second type of structural change is a frequency shift of the $\mathrm{C}=\mathrm{O}$ stretching vibration, due to the perturbation of $\mathrm{p} K_{\mathrm{a}}$ or hydrogen bonding of this group; this would give rise to a derivative-shaped spectral feature in the $1750-1720 \mathrm{~cm}^{-1}$ region. The third type of structural change consistent with the monastrol binding spectrum of the ATPEg5 complex involves these carboxylic acids ligating magnesium, and changes in the ligation of the cation cause a frequency shift of vibrational modes, which can be assigned to the carboxylate ligand (31).

Examination of the Residues Involved in Monastrol and ATP Binding. Future efforts will be directed toward identification of the specific residue(s) in $\mathrm{Eg} 5$ which gives rise to this spectroscopic signature at $1726 \mathrm{~cm}^{-1}$. Perturbation of a carboxylic acid may result in reversible interaction with monastrol, modification of the $\mathrm{Mg}^{2+}$ ligation in the nucleotide binding site, inhibition of ATP hydrolysis, and/or long-range conformation changes affecting polymer binding and force generation. With the goal of establishing candidate carboxylate residues in Eg5, we focus separately on conserved residues across selected kinesins and BimC subfamily members (yellow and blue shaded residues in Figure 4, respectively), as well as the biochemical and molecular data on the broader kinesin, myosin, and G-protein families. Of the sequences shown, including the BimC family, only human (HEg5) and Xenopus Eg5 (XEg5) are known to be sensitive to monastrol $(13,14)$.

To narrow the list of potential candidate residues, we relied on calculations from the CASTp algorithm (32). Two solvent-accessible pockets in the ternary structure of Eg5 were identified (9): one which cradled the MgADP and a second which housed monastrol. Each residue in human Eg5, which contributed at least one atom to the surface of the monastrol binding site (red outline) and of the ADP binding site (green outline), is shown in Figure 4. Residues involved in monastrol binding are not highly conserved between Eg5 and the other members of the BimC subfamily, which is expected, given the specificity of the mitotic inhibitor (13, 14). Candidate carboxylic acids, which give rise to our observed spectroscopic signatures, can be defined on the basis of the information given above. There are 48 carboxylic acid residues in the truncated, human Eg5 utilized in this work. Difference infrared measurements do not preclude measurement of a series of long-distance, propagated, structural changes; however, at first approximation, we favor residues which have potentially reactive, side chain oxygens at the surface of the monastrol- or ADP-binding pockets. These initial candidates are Glu116 in the monastrol-binding pocket (Figure 4, filled red box) and Asp187, Glu201, and Asp322 in the ADP-binding pocket (filled green boxes) of the human Eg5 motor domain.

In contrast to the nonconserved candidate residues gleaned above from the CASTp analysis, biochemical and molecular data on kinesin, myosin, and G-protein families provide other information that needs to be taken into consideration for the conserved carboxylate residue(s), which may be perturbed. For example, in kinesins, myosins, and G-proteins, there are two conserved carboxylic acids in the homologous switch II region. Asp265 in HEg5 is critical for stabilizing the active site $\mathrm{Mg}^{2+}$ ion $(33,34)$, and Glu270 may be involved in a salt bridge with an invariant arginine in switch I $(35,36)$. A conserved Glu345 in the kinesin family has been implicated in phosphate release or conformational transition (37).

Disparity in Changes of Eg5 Secondary Structure upon Binding of Monastrol and the Nucleotide. Spectral components in the infrared amide I region can be assigned to specific forms of secondary structure elements $(38,39)$, based on studies of proteins with known structures $(40-42)$. Infrared analysis is most reliable in the assignment to $\beta$-sheet $(38,42,43)$. Spectral components at $1670-1690 \mathrm{~cm}^{-1}$ and at $1635-1620 \mathrm{~cm}^{-1}$, derived by transition dipole coupling, have also been assigned to $\beta$-sheet (44). When proteins contain predominantly $\beta$-sheet, a maximum around 1634 $\mathrm{cm}^{-1}$ and a strong shoulder at $1660 \mathrm{~cm}^{-1}$ are observed (42); these infrared components are assigned to turns in the protein backbone $(38,39)$.

Assignment of $\alpha$-helical content by IR spectroscopy is not as unequivocal. In the $1630-1620 \mathrm{~cm}^{-1}$ region, potential spectral contributions from both $\alpha$-helices and $\beta$-sheets may overlap. However, assignments between these structures can be discriminated from each other, as $\alpha$-helical structures also contribute an intense spectral component at $1650 \mathrm{~cm}^{-1}$. 
Assignments of $\alpha$-helical structures are most often correlated to this $1650 \mathrm{~cm}^{-1}$ contribution $(45,46)$. In addition, loops or turns can generate spectral components in the vibrational region near $1650 \mathrm{~cm}^{-1}(43,47)$. Infrared contributions near $1640 \mathrm{~cm}^{-1}$ are frequently ascribed to conformational mobility or irregularity in the secondary structure of proteins $(38,39)$.

Given the vibrational studies employed to assign secondary structure to proteins and supporting data from other spectroscopic methods listed above, it is possible to analyze the $1690-1620 \mathrm{~cm}^{-1}$ region of the monastrol binding spectra for net changes in the secondary structure of Eg5. In particular, Eg5-monastrol-ADP samples show changes in this region compared with the Eg5-ADP complex alone: a $(-) 1641 \mathrm{~cm}^{-1}$ line that is normally present in Eg5-ADP samples is replaced by $(+) 1660$ and $1624 \mathrm{~cm}^{-1}$ lines in the presence of monastrol (Figure 3D). These modes are assigned to random and $\alpha$-helical structures, respectively. Therefore, exchange of ADP with Eg5 in the presence of monastrol is associated with a decrease in random structure and a corresponding increase in $\alpha$-helical content. We conclude that binding of nucleotides leads to changes in the secondary structure of Eg5 in the presence of monastrol. These changes observed in the Eg5-monastrol-ADP complex are not restricted to the nucleotide-binding domain. It may be possible, in the future, to utilize selective isotope labeling to attribute the observed changes in secondary structure to specific domains or residues.

The conclusions from our vibrational data are consistent with observations of the crystal structure determination of Yan et al. (9). The motor domain of Eg5, bound with monastrol and $\mathrm{MgADP}$, displayed a number of unique conformational changes in the motor domain (9). An inducedfit pocket for the allosteric inhibitor was generated by movement of the insertion loop (L5, Figure 4). In the presence of monastrol, there is not only a change in the spatial configuration for this insertion loop but also a modification of the regional temperature factor, from relatively high to fairly low. Furthermore, the conserved sequence of switch 1, responsible for nucleotide binding and observed as a loop in the MgADP-Eg5 binary crystal structure $(8)$, is converted to a short helix in the ternary complex (9). Taken together, X-ray diffraction data and the infrared data herein characterize a decrease in conformational mobility and an increase in $\alpha$-helical content in the Eg5monastrol-ADP complex.

It is important to recognize that photolytic release of ATP, versus ADP, leads to a very different set of changes in the secondary structure of Eg5 in the presence of monastrol. In theory, motor proteins must be able to sense a relatively small change in charge localization, the presence of either the $\beta$ or $\gamma$-phosphate, in the active site. In the Eg5-monastrolATP samples, there are two negative lines at 1654 and 1626 $\mathrm{cm}^{-1}$, as well as two positive lines at 1668 and $1637 \mathrm{~cm}^{-1}$ (Figure 3C). These spectral components are assigned to $\alpha$-helical and $\beta$-sheet structures, respectively. Therefore, the Eg5-monastrol-ATP complex is associated with a decrease in $\alpha$-helical content and an increase in $\beta$-sheet content. We propose that the Eg5-ATP complex samples a previously unobserved ternary complex for this mitotic kinesin, one that is modified in the presence of monastrol.

A leading candidate for the structural change we detect upon formation of the Eg5-monastrol-ATP complex is the distal region of switch II (residues 265-270 and helix $\alpha 4$, Figure 4), which is the only $\alpha$-helical site found to be partially disordered in the Eg5-monastrol-ADP crystal structure (9). The switch II helix is a key structural element linking the catalytic site, the polymer-binding site, and mechanical elements of motor proteins. From crystallographic data of G-proteins and kinesins (reviewed in refs 11,12 , and 48), it has been shown that switch II helices are dynamic structures that can access different conformations in a single nucleotide state, and that the region between switch II and its subsequent helix is often disordered. Precedent also exists for the appearance of net $\beta$-sheet content in kinesin. Formation of partial $\beta$ structures and turns has been observed in a kinesin in a complex with AMPPCP and in rat kinesin: switch I assumes a pseudo- $\beta$-hairpin structure, and the neck linker region forms $\beta$-strands that interact with the motor core, respectively (48).

In summary, these results are the first direct observation of dynamic variation in nucleotide-dependent, conformational states of the human Eg5 kinesin motor structure. This application of reaction-induced vibrational spectroscopy delineates conformational changes in the peptide backbone and interactions with substrate phosphate moieties during the nucleotidase cycle in motor proteins. Furthermore, definition of the structural changes at the level of a single amino acid in this work will mark a starting point for future studies, focused on identification of the position and chemical interaction of the carboxylic acid in monastrol inhibition of Eg5. Future insight into this Eg5-monastrol-ATP complex may shed light on the mechanism of energy transduction by Eg5.

\section{ACKNOWLEDGMENT}

We thank Kevin Crosby for generation of the Eg5 expression plasmid and Kenneth Hurley for HPLC analysis of caged nucleotide photolysis. Ross Willis and Dr. Kenneth Meissner were invaluable for their support and efforts in the establishment of the IR and laser systems.

\section{REFERENCES}

1. Mayer, T. U., Kapoor, T. M., Haggarty, S. J., King, R. W., Schreiber, S. L., and Mitchison, T. J. (1999) Small molecule inhibitor of mitotic spindle bipolarity identified in a phenotypebased screen, Science 286, 971-974.

2. Nakazawa, J., Yajima, J., Usui, T., Ueki, M., Takatsuki, A., Imoto, M., Toyoshima, Y. Y., and Osada, H. (2003) A novel action of terpendole $\mathrm{E}$ on the motor activity of mitotic kinesin Eg5, Chem. Biol. 10, 131-137.

3. Hotha, S., Yarrow, J. C., Yang, J. G., Garrett, S., Renduchintala, K. V., Mayer, T. U., and Kapoor, T. M. (2003) HR22C16: a potent small-molecule probe for the dynamics of cell division, Angew. Chem., Int. Ed. 42, 2379-2382.

4. Miki, H., Setou, M., Kaneshiro, K., and Hirokawa, N. (2001) All kinesin superfamily protein, KIF, genes in mouse and human, Proc. Natl. Acad. Sci. U.S.A. 98, 7004-7011.

5. Kashina, A. S., Rogers, G. C., and Scholey, J. M. (1997) The bimC family of kinesins: essential bipolar mitotic motors driving centrosome separation, Biochim. Biophys. Acta 1357, 257-271.

6. Sharp, D. J., McDonald, K. L., Brown, H. M., Matthies, H. J., Walczak, C., Vale, R. D., Mitchison, T. J., and Scholey, J. M. (1999) The bipolar kinesin, KLP61F, cross-links microtubules within interpolar microtubule bundles of Drosophila embryonic mitotic spindles, J. Cell Biol. 144, 125-138.

7. Goshima, G., and Vale, R. D. (2003) The roles of microtubulebased motor proteins in mitosis: comprehensive RNAi analysis in the Drosophila S2 cell line, J. Cell Biol. 162, 1003-1016.

8. Turner, J., Anderson, R., Guo, J., Beraud, C., Fletterick, R., and Sakowicz, R. (2001) Crystal structure of the mitotic spindle kinesin 
Eg5 reveals a novel conformation of the neck-linker, J. Biol. Chem. 276, 25496-25502.

9. Yan, Y., Sardana, V., Xu, B., Homnick, C., Halczenko, W., Buser, C. A., Schaber, M., Hartman, G. D., Huber, H. E., and Kuo, L. C. (2004) Inhibition of a mitotic motor protein: where, how, and conformational consequences, J. Mol. Biol. 335, 547-554.

10. Naber, N., Minehardt, T. J., Rice, S., Chen, X., Grammer, J., Matuska, M., Vale, R. D., Kollman, P. A., Car, R., Yount, R. G., Cooke, R., and Pate, E. (2003) Closing of the nucleotide pocket of kinesin-family motors upon binding to microtubules, Science 300, 798-801.

11. Vale, R. D. (1996) Switches, latches, and amplifiers: common themes of $\mathrm{G}$ proteins and molecular motors, J. Cell Biol. 135 , 291-302.

12. Vale, R. D., and Milligan, R. A. (2000) The way things move: looking under the hood of molecular motor proteins, Science 288, $88-95$.

13. DeBonis, S., Simorre, J. P., Crevel, I., Lebeau, L., Skoufias, D. A., Blangy, A., Ebel, C., Gans, P., Cross, R., Hackney, D. D., Wade, R. H., and Kozielski, F. (2003) Interaction of the mitotic inhibitor monastrol with human kinesin Eg5, Biochemistry 42, 338-349.

14. Maliga, Z., Kapoor, T. M., and Mitchison, T. J. (2002) Evidence that monastrol is an allosteric inhibitor of the mitotic kinesin Eg5, Chem. Biol. 9, 989-996.

15. Kim, S., and Barry, B. A. (2001) Reaction-induced FT-IR spectroscopic studies of biological energy conversion in oxygenic photosynthesis and transport, J. Phys. Chem. B 105, 4072-4083.

16. Cepus, V., Ulbrich, C., Allin, C., Troullier, A., and Gerwert, K. (1998) in Methods in Enzymology (Marriott, G., Ed.) pp 223245, Academic Press, New York.

17. Deavours, B. E., Reddy, A. S., and Walker, R. A. (1998) $\mathrm{Ca}^{2+} /$ calmodulin regulation of the Arabidopsis kinesin-like calmodulinbinding protein, Cell Motil. Cytoskeleton 40, 408-416.

18. Butler, B. C., Hanchett, R. H., Rafailov, H., and MacDonald, G. (2002) Investigating structural changes induced by nucleotide binding to RecA using difference FTIR, Biophys. J. 82, 21982210.

19. Barth, A., Corrie, J., Gradwell, M. J., Maeda, Y., Mantele, W., Meier, T., and Trentham, D. R. (1997) Time-resolved infrared spectroscopy of intermediates and products from photolysis of 1-(2-nitrophenyl)ethyl phosphates: reaction of the 2-nitrosoacetophenone byproduct with thiols, J. Am. Chem. Soc. 119, $4149-4159$

20. Takeuchi, H., Murata, H., and Harada, I. (1988) Interaction of adenosine 5'-triphosphate with $\mathrm{Mg}^{2+}$ : vibrational study of coordination sites by use of ${ }^{18} \mathrm{O}$-labeled triphosphates, J. Am. Chem. Soc. 110, 392-397.

21. Barth, A., and Mantele, W. (1998) ATP-induced phosphorylation of the sarcoplasmic reticulum $\mathrm{Ca}^{2+}$ ATPase: molecular interpretation of infrared difference spectra, Biophys. J. 75, 538-544.

22. Krimm, S., and Bandekar, J. (1986) in Advances in Protein Chemistry (Anfinsen, C. B., Edsall, J. T., and Richards, F. M., Eds.) pp 181-364, Academic Press, New York.

23. Bellamy, L. J. (1980) The Infrared Spectra of Complex Molecules, pp 183-202, Chapman and Hall, London.

24. Dioumaev, A. K., and Braiman, M. (1995) Modeling vibrational spectra of amino acid side chains in proteins: the carbonyl stretch frequency of buried carboxylic residues, J. Am. Chem. Soc. 117 , 10572-10574.

25. Du, X., Frei, H., and Kim, S. H. (2000) The mechanism of GTP hydrolysis by Ras probed by Fourier transform infrared spectroscopy, J. Biol. Chem. 275, 8492-8500.

26. Allin, C., Ahmadian, M. R., Wittinghofer, A., and Gerwert, K. (2001) Monitoring the GAP catalyzed H-Ras GTPase reaction at atomic resolution in real time, Proc. Natl. Acad. Sci. U.S.A. 98 , 7754-7759.

27. Barth, A., Kreutz, W., and Mantele, W. (1990) Molecular changes in the sarcoplasmic reticulum calcium ATPase during catalytic activity. A Fourier transform infrared (FTIR) study using photolysis of caged ATP to trigger the reaction cycle, FEBS Lett. 277, 147-150.

28. Allin, C., and Gerwert, K. (2001) Ras catalyzes GTP hydrolysis by shifting negative charges from $\gamma$ - to $\beta$-phosphate as revealed by time-resolved FTIR difference spectroscopy, Biochemistry 40 , 3037-3046.
29. Cepus, V., Scheidig, A. J., Goody, R. S., and Gerwert, K. (1998) Time-resolved FTIR studies of the GTPase reaction of H-ras p21 reveal a key role for the $\beta$-phosphate, Biochemistry 37, 1026310271

30. Gilbert, S. P., Webb, M. R., Brune, M., and Johnson, K. A. (1995) Pathway of processive ATP hydrolysis by kinesin, Nature 373, 671-676.

31. Nara, M., Torii, H., and Tasumi, M. (1996) Correlation between the vibrational frequences of the carboxylate group and the types of its coordination to a metal ion: an ab initio molecular orbital study, J. Phys. Chem. 100, 19812-19817.

32. Liang, J., Edelsbrunner, H., and Woodward, C. (1998) Anatomy of protein pockets and cavities: measurement of binding site geometry and implications for ligand design, Protein Sci. 7, 18841897.

33. John, J., Rensland, H., Schlichting, I., Vetter, I., Borasio, G. D., Goody, R. S., and Wittinghofer, A. (1993) Kinetic and structural analysis of the $\mathrm{Mg}^{2+}$-binding site of the guanine nucleotide-binding protein p21H-ras, J. Biol. Chem. 268, 923-929.

34. Shimizu, T., Sablin, E., Vale, R. D., Fletterick, R., Pechatnikova, E., and Taylor, E. W. (1995) Expression, purification, ATPase properties, and microtubule-binding properties of the ncd motor domain, Biochemistry 34, 13259-13266.

35. Berchtold, H., Reshetnikova, L., Reiser, C. O., Schirmer, N. K., Sprinzl, M., and Hilgenfeld, R. (1993) Crystal structure of active elongation factor Tu reveals major domain rearrangements, Nature $365,126-132$

36. Fisher, A. J., Smith, C. A., Thoden, J. B., Smith, R., Sutoh, K., Holden, H. M., and Rayment, I. (1995) X-ray structures of the myosin motor domain of Dictyostelium discoideum complexed with $\mathrm{MgADP} \cdot \mathrm{BeFx}$ and $\mathrm{MgADP} \cdot \mathrm{AlF}_{4}$, Biochemistry 34, 89608972.

37. Shimizu, T., Thorn, K. S., Ruby, A., and Vale, R. D. (2000) ATPase kinetic characterization and single molecule behavior of mutant human kinesin motors defective in microtubule-based motility, Biochemistry 39, 5265-5273

38. Surewicz, W. K., Mantsch, H. A., and Chapman, D. (1993) Determination of protein secondary structure by Fourier transform infrared spectroscopy: a critical assessment, Biochemistry 32, 389-394.

39. Jackson, M., and Mantsch, H. H. (1995) The use and misuse of FTIR spectroscopy in the determination of protein structure, Crit. Rev. Biochem. Mol. Biol. 30, 95-120.

40. Susi, H., and Byler, D. M. (1986) Resolution enhanced Fourier transform infrared spectroscopy of enzymes, Methods Enzymol. $130,290-311$

41. de Jongh, H. H., Goormaghtigh, E., and Ruysschaert, J. M. (1996) The different molar absorptivities of the secondary structure types in the amide I region: an attenuated total reflection infrared study on globular proteins, Anal. Biochem. 242, 95-103.

42. Eckert, K., Grosse, R., Malur, J., and Repke, K. R. (1977) Calculation and use of protein-derived conformation-related spectra for the estimate of the secondary structure of proteins from their infrared spectra, Biopolymers 16, 2549-2563.

43. Prestrelski, S. J., Byler, D. M., and Liebman, M. N. (1991) Comparison of various molecular forms of bovine trypsin: Correlation of infrared spectra with X-ray crystal structures, Biochemistry 30, 133-143.

44. Krimm, S., and Bandekar, J. (1986) Vibrational spectroscopy and conformation of peptides, polypeptides, and proteins, Adv. Protein Chem. 38, 181-364.

45. Byler, D. M., and Susi, H. (1986) Examination of the secondary structure of proteins by deconvolved FTIR spectra, Biopolymers $25,469-487$

46. Surewicz, W. K., and Mantsch, H. H. (1988) New insight into protein secondary structure from resolution-enhanced infrared spectra, Biochim. Biophys. Acta 952, 115-130.

47. Wilder, C. L., Friedrich, A. D., Potts, R. O., Daumy, G. O., and Francoeur, M. L. (1992) Secondary structural analysis of two recombinant murine proteins, interleukins 1 alpha and 1 beta: is infrared spectroscopy sufficient to assign structure? Biochemistry $31,27-31$

48. Kull, F. J., and Endow, S. A. (2002) Kinesin: switch I \& II and the motor mechanism, J. Cell Sci. 115, 15-23.

49. Kull, F. J., Sablin, E. P., Lau, R., Fletterick, R. J., and Vale, R. D. (1996) Crystal structure of the kinesin motor domain reveals a structural similarity to myosin, Nature $380,550-555$.

BI048982Y 